\title{
Clinical Audit in Oral Health Care
}

\author{
Shailee Fotedar* \\ Department of Public Health Dentistry, H.P Govt. Dental College, Shimla, India \\ *Corresponding Author: Shailee Fotedar, Department of Public Health Dentistry, \\ H.P Govt. Dental College, Shimla, India.
}

Received: July 29, 2021

Published: August 12, 2021

(C) All rights are reserved by Shailee Fotedar.

\begin{abstract}
Audits are part of the continuous quality improvement process and one of the key elements of clinical governance. Clinical audit includes a detailed review and evaluation of selected clinical records by qualified professional for assessing the quality of dental care. It is 'Measuring and Re-measuring' against agreed standard for improvement. Clinical audit in oral Health care at present is only sporadic in India. The present review article aims to introduce the concept of clinical audit and it should be conducted on regular basis in oral health care. The various health organisations and associations can play a pivotal role by increasing the awareness among the health professionals about clinical audit.
\end{abstract}

Keywords: Audit; Oral Health Care and Clinical

\section{Introduction}

Audit can be defined as a hearing; especially a judicial examination of complaints or an official examination of accounts with verification by reference to witnesses and vouchers, or a critical evaluation [1]. It has been defined as "the systematic appraisal of the implementation and outcome of any process in the context of prescribed targets and standards" [2].

The term Audit has been included in different settings (business, health care etc.) for methods which aim to ensure that the procedures carried out for a purpose are consistent and effective for the achievement of objectives. The term 'clinical audit' is used to describe a process of assessing clinical practice against standards. The Commission on Patient Safety and Quality Assurance defined clinical audit as 'a clinically led, quality improvement process that seeks to improve patient care and outcomes though the systematic review of care against explicit criteria and to act to improve care when standards are not met' [3]. The main aim of Clinical (or medical) audits is the continuous quality improvement on specific issues or aspects of health care and clinical practice.
Auditing patient care is a centuries old concept, that is progressively acquiring importance as a potential device to reduce morbidity and mortality, and thus improve quality of life. Historically, audit has been recorded as early as in 1066 in Domesday Book with the development of national statistics of births and deaths [4]. In 1750 BC, King Hammurabi of Babylon penalized clinicians on poor performance in order to ensure adequate patient care [5,6]. Florence Nightingale is considered as the pioneer of clinical audit, as her assessment of the effectiveness of cleanliness and its enforcement resulted in tremendous reduction in mortality rates of hospitalized patients during the Crimean war of 1853-5. Ernest Codman is recognized as being the first true medical auditor for his work in 1912 on monitoring surgical outcomes of his patients [5-8]. In recent years, the concept saw light when the United Kingdom pioneered the move to integrate clinical audit in professional healthcare with the introduction of the white paper [5,7].

\section{Importance}

Clinical audits are a quality improvement tool and form one of the seven pillars of clinical governance [9]. The tool aids in identify- 
ing areas of practice which require improvement when compared to accepted standards. While highlighting any areas which may require improvement, it also identifies and reinforces areas of good practice [10]. It acts as an essential tool for quality improvement and assurance of the safe and efficient operation of any clinic in order to sustain and maintain a greater standard of patient care [11].

In other words, clinical audit means:

- $\quad$ Looking at how well we currently perform something

- $\quad$ Setting a target for how well we want to do it

- $\quad$ Deciding how we will make the improvement putting this in place

- $\quad$ Measuring again to see if we have achieved the target [12].

Clinical Audit (CA) is about identifying the areas of potential improvement and accordingly putting steps in place time to time. Thus, it becomes a process of continuous improvement in quality of service provision to patients. Owing to the cyclical nature of the CA and the dynamism it brings to a healthcare organization, CA is often referred as 'vital signs' or 'pulse' of the organization [13]. The targets for improvement that services set themselves should reflect their stated aims as well as the areas currently requiring extra attention.

Clinical audit process is known as the 'Audit cycle' $[9,11,14,15]$.

\section{Types of clinical audit}

Lokuarachchi in 2006 [16] gave three different types of audits:

1. Standards-based audits.

2. Adverse occurrence screening and critical incident monitoring.

3. Peer-review.

\section{Standards-based audits}

This type of audit involves collecting data regarding current practice and comparing them to defined standards. This allows any areas requiring improvement to be highlighted and addressed, bringing necessary changes into practice.

Adverse occurrence screening and critical incident monitoring

This type of audit would be undertaken to evaluate occurrences with unanticipated results or special concern. It allows reflection of the dental team's performance and offers knowledge for future application.

\section{Peer review}

Individual cases are discussed by peers to determine whether best care was given. Somewhat similar to type 2 above but might include interesting or unusual cases rather than critical incidents. Recommendations are very often not followed as there is no systematic method to follow. Peer review is recommended by the Regulation of Dental Services Programme Board as a method of preventing the emergence of poor practice and allowing the maintenance of good practice. The Care Quality Commission (CQC) deems peer review to be one of the hallmarks of well-led practice. Benefits of peer review include the sharing of knowledge between colleagues, individual learning and a method to support daily practice.

Further to the above, audits can be either prospective or retrospective. Prospective audits permit collection of real-time data reflecting the current process of care. This type of audit is timelimited and provides more precise data, however increased awareness of the ongoing audit may impact performance. Retrospective audits act as a historical benchmark of practice; they can span a much longer time period and are not subject to corruption by the audit process [17].

\section{Clinical Audit Cycle}

Stage 1 - Planning for audit: This involves planning for the audit, including choosing a team, co-ordinator and a topic. Ideally, the topic should be relevant with potential benefit to patient care or the organisation. If a clinical audit is to be successful in identifying areas of excellence or areas for improvement, it requires effective planning and preparation. The amount of planning and preparation will depend on the specific circumstances of each audit. Planning for audit can be described in three main steps:

1. Step 1: Involving stakeholders: anyone involved in providing or receiving care can be considered a stakeholder in clinical audit.

2. Step 2: Determining the audit topic.

3. Step 3: Planning the delivery of audit fieldwork. 
Any topic which is selected for clinical audit - perhaps an investigation, treatment or procedure - should be chosen on the basis of its relevance to improve the patient outcomes and not to satisfy the personal curiosity. Clinical procedures, risk management protocols, cross - infection control mechanisms, staff management and responsibilities, implementation of quality assurance protocols, monitoring and evaluation systems should be analysed. This involves all the steps involved from the point a patient is received in the practice till he is discharged after treatment. Examples can be taking proper clinical notes and keeping a record of them; having a system for recording the complaints - verbal and written- by the patients and reviewing these complaints in the dental practice meetings, with the objective of implementing any changes in the practice to minimise future complaints; recording all adverse events cases to minimise the risk of any such event occurring in the future; and havingcontinuing professional development training in place for the staff [18].

Stage 2 - Define standards and criteria: Criteria are explicit statements that define what is being measured. It is a measurable outcome of care. A criterion is used to assess quality of care and is preferably evidence based; therefore a literature search is essential [19]. Criteria are often also called guidelines or benchmarks and are based on biomedical research and health technology assessment. They are outcome orientated and usually developed by a multidisciplinary team [20].

Standards: Standards specify what should be provided and how. They should be developed from the evidence of best practice by looking at specific areas of care. Audit standards must be SMART.

- $\quad$ Specific covers one topic only.

- Measurable can be measured in a practical way.

- Achievable is something that is reasonable for staff to achieve.

- Relevant is an issue that is important to patients and staff.

- $\quad$ Timescale can be measured within a reasonable period of time.

This step involves defining and selecting the criteria and standards to which the performance will be compared. Unfortunately, the quality of published guidelines is variable, many not being explicit or evidence based [18]. If no acceptable guidelines are available, the organisation needs to develop their own best practice guidelines before the clinical audit is commenced.

Stage 3 - Measuring performance: This stage may be described in steps as follows:

1. Step 1: Data collection: The data should be precise and relevant, and collected over a specified time period. Patient registers may be used to identify patients, and clinical records are often used as data sources. Audit staff needs to be careful about the accuracy, timeliness and completeness of clinical records. The data collected must be adequate (sample size) and relevant to enable making valid conclusions [21]. If a questionnaire is used, it should be clear, concise and unambiguous, avoiding double-barrelled and leading questions. Data are generally collected retrospectively and most audits only require the use of basic descriptive statistics [22]. The data on patient information has to be used as per Caldicott Principles given by Caldicott Committee in 1996.These include:

- Justify the purpose

- Don't use patient-identifiable information unless it is absolutely necessary

- Use the minimum necessary patient-identifiable information

- The access to the patient-identifiable information should be on a strict need-to-know basis

- $\quad$ Everyone should be aware of their responsibilities

- $\quad$ Understand and comply with the law [23].

2. Step 2: Data analysis: Convert a collection of data into information in order to identify the level of compliance with the agreed standard. Where possible, the results should be compared with audits performed by peer groups or national standards [24].

3. Step 3: Drawing conclusions: Identify the reason why standard was not met. 
4. Step 4: Presentation of results: The impact of clinical audit on audience should be maximised in order to generate discussion and to stimulate and support action planning.

\section{Stage 4 - Making improvements}

As the main purpose of performing clinical audit is to find out the degree to which the clinical services provided comply with the accepted evidence based practice standard. Clinical audit results may give us excellent areas of or practice which needs to be appreciated. Andin the audit summary report, we need to make a statement saying 'no further action required' and a rationale why re-audit is not required. Alternatively Clinical audit results may also give us 'areas for improvement' where the required standards are not being met. So, the clinical audit group should discuss the findings in order to clarify the areas where action is required in order to improve the quality of clinical care and its outcomes. Where audit has shown that there are serious concerns regarding the practice of an individual, these should be conveyed immediately to the audit sponsor, who should inform the manager of the service for urgent action [25].

\section{Stage 5 - Sustaining improvements}

The audit cycle is a continuous process. According to Ashmore, Ruthven and Hazelwood 'A complete audit cycle ideally involves two data collections and a comparison of one with the other, following implementation of change after the first data collection, in order to determine whether the desired improvements have been made. Further cycles may be necessary if performance still fails to attain the levels set at the outset of the audit. At this stage there may be justification for adjusting the desired performance levels in the light of the results obtained.' Where quality improvement plans are put in place, monitoring should be performed to ensure plans are implemented as agreed and within the agreed time frame. Clinical leads/or managers who agree to implement quality improvement plans are accountable for the delivery of quality improvement plans and sustaining quality improvement. A summary report of progress should be submitted through the appropriate lines of responsibility at regular intervals [26].

\section{Re-auditing}

Audit is a continuous cycle. If following an initial audit it is found that desired performance levels are not being reached, and a pro- gramme of change activity has been put in place, then the audit should be repeated to show whether the changes implemented have improved care or whether further changes are required. This cycle is repeated until the desired performance levels are being achieved [27].

Example: Longevity of fissure sealants can be audited in a following manner:

Background: In order to prevent caries progression, the fissure sealants must be intact.

Defining standards and criteria: At least 95\% should be intact after six months.

\section{Measuring performance:}

1. Data collection: No of the teeth fissure sealed. And the number of teeth having intact fissure sealants at the end of six months (minimum number of teeth to be sealed 100120).

2. Data analysis: The data collected needs to be analysed and compared with the standard.

Draw conclusions: Identify the reason why sealant failed and discuss ways to improve the technique of application.

Re Audit: Annually.

Indian scenario: At present there are only sporadic audits in India, indicating the recognition for monitoring of quality health care services. So, an action plan from various oral health care organisations and associations must be formulated for the Indian setup. The CDE programs can be arranged to create awareness among the health care providers throughout the country regarding the importance of clinical audit. Clinical Audit tool and audit model developed for oral healthcare should be relevant to the Indian setting.

\section{Conclusion}

Audits are part of continuous quality improvement in any health care and one of the key elements of clinical governance. Clinical Audits compare current practice against an accepted standard that has been set and examine whether this practice meets the standard. Clinical Audit is a cyclical process: it compares practice to standards, measures performance, makes improvements 
and, importantly, involves a re-audit after a time period to ensure that the improvement is sustained. At present in India there are only sporadic Clinical Audits. So various health organisations and

\section{Bibliography}

1. Frostick SP., et al. "Medical audit: Rationale and Practicalities". Cambridge: Cambridge University Press (1993).

2. Gregg TA and Boyd DH. "A computer software package to facilitate clinical audit of outpatient paediatric dentistry". International Journal of Paediatric Dentistry 6 (1996): 45-51.

3. National Institute for Clinical Excellence, CHI, Royal College of Nursing, University of Leicester. "Principles for Best Practice in Clinical Audit". Oxon, UK: Radcliffe Medical Press Ltd (2002): 1-9.

4. Frostick SP., et al. "Medical audit: Rationale and Practicalities". Cambridge: Cambridge University Press (1993).

5. Lokuarachchi SK. "Clinical Audit-What is it and how to do it?" Galle Medical Journal 11 (2006): 41-43.

6. Copeland G. "NHS Clinical Governance Support Team". London: Department of Health publications; 2005. A Practical Handbook for Clinical Audit (2005).

7. Clinical audit, From Wikipedia, the free encyclopedia (2011).

8. Ashwini NS., et al. "Basics in research methodology: The clinical audit”. Journal of Clinical and Diagnostic Research 5 (2011): 679-682.

9. Mikhaeel G. "Reinvigorating Clinical Audit in a Major Teaching Hospital”. GSTT Clinical Audit. UK: Guy's and St Thomas NHS Foundation Trusts (2007).

10. Choudhary CR. "Engaging the element of clinical governance: quality dental care services in India". Indian Journal of Dental Research 4 (2010): 1e4.

11. Jenkins C. "Carrying Out Clinical Audit at Queen Victoria Hospital, Clare (2009).

12. Selman L and Harding R. "How can we improve outcomes for patients and families under palliative care? Implementing clinical audit for quality improvement in resource limited settings". Indian Journal of Palliative Care 16.1 (2010): 8e15.
13. Bullock R. "Clinical governance, the emperor's new clothes and the meaning of clinical audit". Journal of Clinical Excellence 4 (2002): 43e46.

14. Cannell P. "Clinical Audit and Dentistry". University of Essex, UK.

15. Schonfeld HK., et al. "The content of good dental care: methodology in a formulation for clinical standards and audits, and preliminary findings". American Journal of Public Health 57 (1967): 1137e1146.

16. Lokuarachchi S K. "Clinical audit - what is it and how to do it?" Galle Medical Journal 11 (2006): 41-43.

17. Clinical Governance Support Team. A practical handbook for clinical audit (2005).

18. Holden LC and Moore RS. "The development of a model and implementation process for clinical governance in primary dental care". The British Dental Journal 196 (2004): 21-24.

19. Verma R. "Clinical audit". Anaesthesia and Intensive Care: SAGE Journals 5 (2004): 410-412.

20. Oosterhuis W., et al. "Evidence-based guidelines and principles in laboratory medicine: principles and methods". Clinical Chemistry 50 (2004): 806-818.

21. Verma R. "Clinical audit". Anaesthesia and Intensive Care: SAGE Journals 5 (2004): 410-412.

22. Oosterhuis W., et al. "Evidence-based guidelines and principles in laboratory medicine: principles and methods". Clinical Chemistry 50 (2004): 806-818.

23. Kraszewski Sarah. "Clinical audit in general practice". Practice Nurse 30.10 (2005): 51-55.

24. Verma R. "Data quality and clinical audit". Anaesthesia and Intensive Care: SAGE Journals 7 (2006): 342-344.

25. Erasmus R T and A E Zemlin. "Clinical audit in the laboratory". Journal of Clinical Pathology 62 (2009): 593-597.

26. Buttery Y. "Implementing evidence through clinical audit. Evidence-based healthcare". Oxford: Butterworth-Heinemann (1998): 182-207. 
Clinical Audit in Oral Health Care

27. Dixon N. "Getting clinical audit right to benefit patients". Getting Clinical Audit Right (2007).

Volume 5 Issue 9 September2021

(c) All rights are reserved by Shailee Fotedar. 